\title{
COX2 Positive
}

National Cancer Institute

\section{Source}

National Cancer Institute. COX2 Positive. NCI Thesaurus. Code C150490.

An indication that COX2 expression has been detected in a sample. 